\title{
Treatment option of endoscopic stent insertion or gastrojejunostomy for gastric outlet obstruction due to gastric cancer: a propensity score-matched analysis
}

\author{
Yoshio Haga ${ }^{1}$ (1) $\cdot$ Naoki Hiki $^{2} \cdot$ Takahiro Kinoshita $^{3} \cdot$ Toshiyasu Ojima $^{4} \cdot$ Yoshihiro Nabeya $^{5} \cdot$ Shirou Kuwabara $^{6}$. \\ Yasuyuki Seto ${ }^{7} \cdot$ Kazuhito Yajima $^{8} \cdot$ Hiroya Takeuchi $^{9} \cdot$ Kazuhiro Yoshida $^{10} \cdot$ Yasuhiro Kodera $^{11} \cdot$ Yoshiyuki Fujiwara $^{12}$. \\ Hideo Baba ${ }^{13}$
}

Received: 2 June 2019 / Accepted: 10 January 2020 / Published online: 25 January 2020

(c) The International Gastric Cancer Association and The Japanese Gastric Cancer Association 2020

\begin{abstract}
Background There are currently two treatment options for gastric outlet obstruction (GOO) due to gastric cancer, endoscopic stenting and surgical gastrojejunostomy. However, their therapeutic effects have not yet been established. Therefore, the present study was undertaken to examine these effects.

Methods The Japanese Gastric Cancer Association invited its delegates to participate in a retrospective multicenter cohort study on patients with GOO due to gastric cancer who underwent stent therapy or gastrojejunostomy in 2015.

Results We obtained data from 85 patients undergoing stent therapy and 94 undergoing gastrojejunostomy from 42 hospitals. Baseline data revealed that stent patients had lower food intake, poorer performance status, and worse prognostic indices than gastrojejunostomy patients. Postoperative food intake and survival times were worse in stent patients than in gastrojejunostomy patients. We performed propensity score matching to select pairs of patients with similar baseline characteristics in the two treatment groups. After matching, the frequency of postoperative complications was significantly less in stent patients $(3 \%, 1 / 33)$ than in gastrojejunostomy patients $(21 \%, 7 / 34 ; p=0.03)$. A low residue or full diet was achieved by $97 \%$ of stent patients (32/33) and 97\% of gastrojejunostomy patients (33/34) $(p=0.98)$. Median survival times were 7.8 months in stent patients and 4.0 months in gastrojejunostomy patients $(p=0.38)$.

Conclusions Propensity score matching demonstrated that endoscopic stent placement resulted in less postoperative morbidity than and a similar food intake and equivalent survival times to gastrojejunostomy. These results suggest the utility of stent therapy.
\end{abstract}

Keywords Gastric carcinoma · Gastric outlet obstruction · Stent · Gastrojejunostomy

\section{Introduction}

Gastric cancer is the third leading cause of cancer death worldwide [1]. Multimodality therapies for advanced gastric cancer, including surgery, chemotherapy, targeted therapy, immunotherapy, and radiation therapy, have been shown to improve survival times [2]. Surgical resection for

Electronic supplementary material The online version of this article (https://doi.org/10.1007/s10120-020-01040-0) contains supplementary material, which is available to authorized users.

Yoshio Haga

haga-yoshio@amakusa.jcho.go.jp

Extended author information available on the last page of the article tumors that were originally unresectable, but became resectable after chemotherapy has been advocated as "conversion therapy" to achieve a cure [3]. Gastric outlet obstruction (GOO) is one of the most detrimental features of advanced distal gastric cancer [4-11]. It reduces the quality of life, nutritional status, and performance status of patients and, hence, deprives them of the opportunity for proper treatment. Therefore, the treatment of GOO plays a key role in the management of obstructive unresectable distal gastric cancer.

There are currently two treatment options to alleviate GOO due to unresectable gastric cancer: endoscopic metallic stent insertion and surgical gastrojejunostomy [4-11]. Although gastrojejunostomy was previously performed as open hand-sewn surgery, recent advances in surgical tools 
have enabled laparoscopic surgery in this field. Delayed gastric emptying (DGE) is an adverse event of gastrojejunostomy and its incidence was previously reported to be between 10 and 26\% [9]. Stomach partitioning and Braun anastomosis, side-to-side jejunojejunostomy between the afferent and efferent loops, have been suggested to prevent DGE $[5,9]$. Regarding stent therapy, technical success, defined as the successful placement of a stent, was reported in $100 \%$ of cases at a high-volume center [8]. Clinical success, defined as the significant alleviation of symptoms, was achieved in $77.4 \%$ of cases [8]. Stent obstruction is sometimes encountered in stent therapy, mostly due to tumor ingrowth, and requires reintervention [8].

Previous studies revealed that stent therapy was more likely to be selected for advanced and physically deteriorated patients, and gastrojejunostomy for more active patients [4-11]. However, due to the rarity of GOO due to gastric cancer, these studies did not have sufficient sample sizes. It currently remains unclear which treatment will be more beneficial for patients with GOO due to gastric cancer. Furthermore, indications for these therapies have not yet been established. Therefore, there are currently no recommendations for the treatment of GOO due to gastric cancer. The present study was undertaken to elucidate the current status of these treatments via a nationwide survey in Japan and to examine their therapeutic effects.

\section{Patients and methods}

\section{Study design}

This was a multicenter retrospective cohort study. The study protocol was approved by the Ethical Review Board of the Japanese Gastric Cancer Association (JGCA) on Dec 4, 2015. JGCA invited its delegates to participate in this study via a surface mail letter. The study protocol was also approved by the Institutional Review Board of each participating hospital.

The present study was performed based on the Ethical Guidelines for Medical and Health Research Involving Human Subjects in Japan [12]. All data were abstracted from medical records from which personal identifiers were redacted. Informed consent from patients was waived, but information on this study was disclosed in the study website of each participating hospital during data collection. The study protocol was registered with the Japanese official registry site, University Hospital Medical Information Network (UMIN) on Oct 29, 2017 (UMIN000029732), before the collection of patient data. A summary of the study protocol is available at https://upload.umin.ac.jp/cgi-open-bin/ctr/ ctr_view.cgi?recptno=R000033633.

\section{Patients}

The inclusion criterion for the present study was patients who underwent endoscopic stenting or gastroenterostomy for stenosis of the gastric pylorus or duodenum due to gastric cancer between January 2015 and December 2015. The exclusion criterion was patients who had non-epithelial tumors.

\section{Data collection}

Data were retrospectively collected on the preoperative physiological status, laboratory data, tumor status, details of interventions, postoperative complications, the gastric outlet obstruction scoring system (GOOSS) before and after interventions, and postoperative overall survival times. GOOSS is defined as 0 for no oral intake, 1 for liquids only, 2 for soft solids, and 3 for a low residue or full diet [13]. Tumor-related factors were classified according to the general rules for gastric cancer [14]. Postoperative complications were defined as any adverse events occurring within 30 days of the Clavien-Dindo classification two or greater [15].

We assessed various prognostic indices. The Glasgow prognostic score (GPS) is a cumulative inflammation-based cancer prognostic marker composed of serum levels of C-reactive protein and albumin [16]. Onodera's prognostic nutritional index (PNI) is a simple index that consists of serum albumin levels and total lymphocyte counts in peripheral blood [17]. The preoperative risk score (PRS) of the E-PASS scoring system is composed of age, the performance status (PS), American Society of Anesthesiologists Physical Status (ASA-PS), and the comorbidities of diabetes mellitus, severe heart disease, and severe pulmonary disease [18].

\section{Statistical analysis}

All statistical analyses were performed using the Stata 14.2 (Stata Corp., College Station, TX, USA) software program. Two-tailed $p$ values $<0.05$ were considered to be significant.

Continuous variables between groups were compared using the Wilcoxon rank-sum test. Categorical variables between groups were compared using the $\chi^{2}$ test with Yates correction for continuity where appropriate. Univariate and multivariate analyses to examine the probability of binary variables were performed using a logistic regression analysis. Postoperative overall survival rates were calculated using the Kaplan-Meier method. Univariate and multivariate analyses for survival were performed using Cox's regression analysis. 
The propensity score, defined as the conditional probability of receiving treatment, was created by a logistic regression analysis using pretreatment characteristics as independent variables [19]. The discriminatory power of the propensity score for the assignment of treatment was measured by the area under the receiver-characteristic curve (AUC). AUC ranges between 0.5 and 1.0, with a higher AUC indicating better discriminatory power. We then performed nearest neighbor matching within specified caliper widths to select two treatment groups with similar characteristics [19].

\section{Results}

\section{Baseline characteristics}

The secretariat of the JGCA sent invitation letters to 326 delegates at 212 hospitals. Seventy-seven hospitals declared their participation, and data were ultimately provided by 42. We obtained data on 85 patients who underwent stent therapy and 94 who underwent gastrojejunostomy. The baseline data of these patients are shown in Table 1. The stent group had lower GOOSS, poorer performance status and worse prognostic indices. Of note, the ratio of PNI score greater than 40 , which had been implicated as a risk factor of postoperative complications [17], was significantly higher in stent group $(56 \%, 53 / 94)$ than that in gastrojejunostomy group $(24 \%, 20 / 85 ; p<0.001)$. Similarly, the ratio of PRS $\geq 0.5$, which had been reported as a risk factor of postoperative complications [18], was significantly higher in stent group $(55 \%, 47 / 85)$ than that in the gastrojejunostomy group $(29 \%, 27 / 94 ; p<0.001)$. More than one-fourth $(27 \%)$ of the gastrojejunostomy group had preoperative GOOSS of 3 and a low residue or full diet before surgery, in contrast to only $11 \%$ of the stent group. Most of the gastrojejunostomy group (88\%) and only approximately half of the stent group (54\%) had a good performance status of 0 or 1 . Macroscopic type IV was more frequent in the stent group.

\section{Clinical outcomes of all patients}

Regarding interventions, metallic stent placement was performed on 55 patients using the Niti-S® type of Taewoong Medical Co. Ltd., Goyang, South Korea, on 27 using the WallFlex ${ }^{\circledR}$ type of Boston Scientific Co. Ltd., Natick, MA, USA, on two using Evolution ${ }^{\circledR}$ of Cook Medical, Bloomington, IN, USA, and on one using an undocumented product. Covered and uncovered stents were placed in 22 and 63 patients, respectively. Technical success was achieved in $99 \%$ of the stent group (84/85). Regarding gastrojejunostomy, open surgery was performed on 47 patients and laparoscopic surgery on 47 . Mechanical suture was performed on 84 patients and hand suture on 10 . Antecolic reconstruction
Table 1 Baseline data for all patients

\begin{tabular}{|c|c|c|c|}
\hline & $\begin{array}{l}\text { Stent group } \\
(n=85)\end{array}$ & GJ group $(n=94)$ & $p$ value \\
\hline Age & $71(27-92)$ & $71(36-94)$ & 0.90 \\
\hline Sex, $\%$ male & $68 \%$ & $68 \%$ & $>0.99$ \\
\hline Body mass index & $\begin{array}{l}20(14-30) \\
(n=77)\end{array}$ & $\begin{array}{l}20(12-32) \\
(n=94)\end{array}$ & 0.18 \\
\hline GOOSS, $0: 1: 2: 3$ & 39: $25: 12: 9$ & 17: $33: 19: 25$ & $<0.001$ \\
\hline PS, 0: 1: 2: 3: 4 & 8: $38: 22: 16: 1$ & 43: 40: 9: 2: 0 & $<0.001$ \\
\hline ASA-PS, 1: 2: 3 & 9: $60: 16$ & 21: $61: 12$ & 0.08 \\
\hline GPS, $0: 1: 2$ & $\begin{array}{l}5: 40: 37 \\
(n=82)\end{array}$ & $\begin{array}{l}37: 35: 21 \\
(n=93)\end{array}$ & $<0.001$ \\
\hline PNI & $\begin{array}{l}35(16-52) \\
(n=75)\end{array}$ & $\begin{array}{l}42(27-54) \\
(n=89)\end{array}$ & $<0.001$ \\
\hline PRS & $0.57(0.13-1.0)$ & $0.43(0.12-0.92)$ & $<0.001$ \\
\hline $\begin{array}{l}\text { Macroscopic type } \\
\text { IV }\end{array}$ & $37 \%(30 / 82)$ & $10 \%(9 / 94)$ & $<0.001$ \\
\hline cT stage, $2: 3: 4$ & $\begin{array}{l}3: 40: 39 \\
(n=82)\end{array}$ & $\begin{array}{l}2: 35: 57 \\
(n=94)\end{array}$ & 0.19 \\
\hline $\mathrm{cN}(+)$ & $81 \%(69 / 85)$ & $91 \%(86 / 94)$ & 0.043 \\
\hline Distant metastasis & $79 \%(67 / 85)$ & $69 \%(65 / 94)$ & 0.14 \\
\hline Liver & $31 \%(26 / 85)$ & $20 \%(19 / 94)$ & 0.11 \\
\hline Peritoneum & $53 \%(45 / 85)$ & $48 \%(45 / 94)$ & 0.50 \\
\hline $\begin{array}{l}\text { Distant lymph } \\
\text { nodes }\end{array}$ & $16 \%(14 / 85)$ & $19 \%(18 / 94)$ & 0.64 \\
\hline Other organs & $7 \%(6 / 85)$ & $5 \%(5 / 94)$ & 0.63 \\
\hline CEA, ng/mL & $\begin{array}{l}4.8(0.5-2828) \\
(n=77)\end{array}$ & $\begin{array}{l}4.0(0-5387) \\
(n=93)\end{array}$ & 0.08 \\
\hline
\end{tabular}

Data for continuous variables are shown as medians (range)

$p$ values less than 0.05 are highlighted by bold letters

GJ gastrojejunostomy, GOOSS gastric outlet obstruction scoring system, 0 for no oral intake, 1 for liquids only, 2 for soft solids, 3 for a low residue or full diet, $P S$ performance status, ASA-PS American Anesthesiologists Association Physical Status, GPS Glasgow Prognostic Score, PNI Prognostic Nutritional Index, PRS Preoperative Risk Score

was performed on 81 patients, retrocolic reconstruction on 4 , and undocumented on 9. Stomach partitioning was performed on 54 patients (57\%) and Braun anastomosis on 32 $(34 \%)$.

Table 2 shows outcome data. Postoperative complications were less common in the stent group than in the gastrojejunostomy group. In stent group, postoperative complications included one case of pleural effusion, two cases of pneumonia, one case of cerebral stroke, one case of deep vein thrombosis, one case of respiratory failure, and one case of tumor bleeding occurred in 3 patients. Two of them died in hospital. In gastrojejunostomy group, postoperative complications included two cases of pneumonia, two cases of sepsis, one case of intra-abdominal abscess, one case of small bowel obstruction, one case of urinary tract infection, one case of gastrointestinal bleeding, one case of progressive renal dysfunction, and 6 cases of other complications 
Table 2 Outcome data for all patients

\begin{tabular}{lllc}
\hline & Stent group $(n=85)$ & GJ group $(n=94)$ & $p$ value \\
\hline Postoperative complications & $4 \%(3 / 85)$ & $14 \%(13 / 94)$ & $\mathbf{0 . 0 2}$ \\
In-hospital mortality & $13 \%(11 / 85)$ & $6 \%(6 / 94)$ & 0.14 \\
Length of stay, days $^{\mathrm{b}}$ & $27(2-147)$ & $21(7-467)$ & 0.20 \\
& $(n=85)$ & $(n=94)$ & \\
Clinical success $^{\mathrm{a}}$ & $87 \%(74 / 85)$ & $69 \%(65 / 94)$ & $<\mathbf{0 . 0 0 1}$ \\
GOOSS 3 achieved $^{\text {Duration of GOOSS 3, days }}{ }^{\mathrm{b}}$ & $81 \%(69 / 85)$ & $95 \%(89 / 94)$ & $\mathbf{0 . 0 2}$ \\
& $96(2-616)$ & $182(10-997)$ & $\mathbf{0 . 0 0 1 2}$ \\
Induction of chemotherapy & $(n=55)$ & $(n=72)$ & 0.07 \\
Subsequent resection & $51 \%(43 / 85)$ & $64 \%(60 / 94)$ & $<\mathbf{0 . 0 0 1}$ \\
Survival time, months & $1 \%(1 / 85)$ & $15 \%(14 / 94)$ & $<\mathbf{0 . 0 0 1}$ \\
& $4.3(1.6-8.5)$ & $7.9(2.8-19)$ & $(n=92)$ \\
\hline
\end{tabular}

GJ gastrojejunostomy, GOOSS Gastric Outlet Obstruction Scoring system, 0 for no oral intake, 1 for liquids only, 2 for soft solids, 3 for a low residue or full diet.

andicates an improvement in GOOSS [4].

${ }^{\mathrm{b}}$ Data represent medians (range)

${ }^{c}$ Data represent medians (interquartile range). $P$ values less than 0.05 are highlighted by bold letters occurred in 13 patients. Three of the patients died in hospital. DGE occurred in 1 patient in the stent group $(1 \%, 1 / 85)$ and in 2 in the gastrojejunostomy group (2\%, 2/94). Regarding gastrojejunostomy, open surgery was performed on the 2 patients who developed DGE; one patient had stomach partitioning without Braun anastomosis, while the other had Braun anastomosis without stomach partitioning. Clinical success was more frequently achieved in the stent group than in the gastrojejunostomy group. This result was attributed to one-fourth of the gastrojejunostomy group already having GOOSS 3 before surgery. GOOSS 3 was achieved in $81 \%$ of the stent group and in $95 \%$ of the gastrojejunostomy group. In the stent group, stent obstruction occurred in 16 patients (19\%) until death. Stent obstruction occurred in 17\% (11/63) of uncovered stent patients and in 22\% (5/22) of covered stent patients. Most patients recovered following the stentin-stent technique $(94 \%, 15 / 16)$. More than $50 \%$ of patients in both groups subsequently received chemotherapy. Subsequent resection was more uncommon in the stent group (only in one patient who showed no recurrence for more than two years). Postoperative survival times were significantly longer in the gastrojejunostomy group (Fig. 1a).

\section{Multivariate analysis}

We examined prognostic factors to achieve GOOSS 3 in all patients (Table 3). A univariate analysis was initially performed to investigate relationships between individual factors and postoperative GOOSS 3 . We identified 8 significant factors: macroscopic type IV, cT stage, preoperative GOOSS, PS, ASA-PS, serum Na levels, CEA levels, and the selection of stenting or gastrojejunostomy. A multivariate analysis using these variables revealed that macroscopic type
IV and the performance status were independent prognostic factors to achieve GOOSS 3 . The selection of stenting or gastrojejunostomy was not an independent risk factor. When we selected patients with PS 2 or less having no macroscopic type IV tumors, GOOSS 3 was obtained in 97\% (37/38) of the stent group and in $94 \%$ (78/83) of the gastrojejunostomy group $(p=0.43)$.

We then investigated prognostic factors for postoperative overall survival (Table 4). A univariate analysis was initially performed to examine the relationships between individual factors and survival. We identified 11 significant factors: age, macroscopic type IV, tumor location of middle part involvement, preoperative GOOSS, PRS, biliary stent, GPS, serum Na level, hemoglobin level, PNI, and the selection of stenting or gastrojejunostomy. A multivariate analysis using these variables revealed that macroscopic type IV and PRS were independent prognostic factors for survival. The selection of stenting or gastrojejunostomy was not an independent prognostic factor. When we selected patients with PRS $\leq 0.6$ and no macroscopic type IV tumors, median survival times were similar between the groups; 7.8 months for the stent group $(n=30)$ and 9.2 months for the gastrojejunostomy group $(n=66) ; p=0.21$.

\section{Evaluation by propensity score matching}

We performed propensity score matching to reduce selection bias. The propensity score we made showed a high discriminatory power to predict the selection of interventions, the AUC of which was 0.91. After matching, baseline data were balanced (Table 5). Outcome data after matching revealed that postoperative complications were significantly less frequent in the stent group (Table 6). In 


\section{a Before propensity score matching}

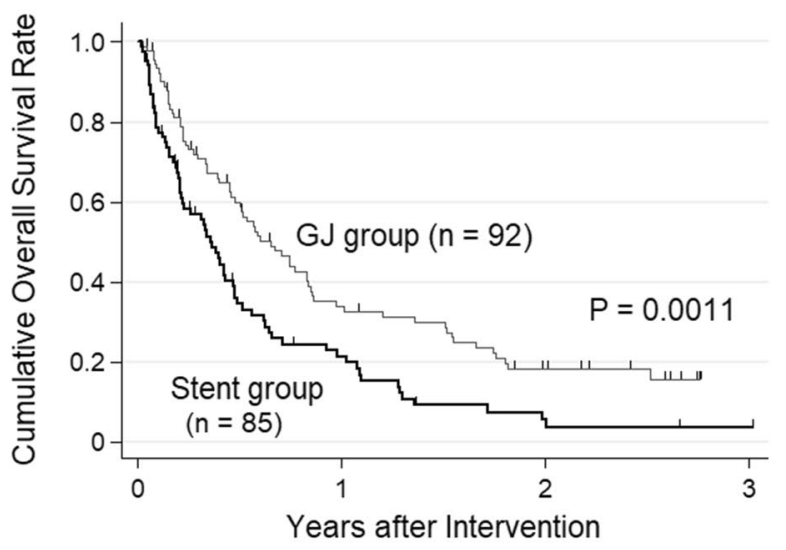

\section{b After propensity score matching}

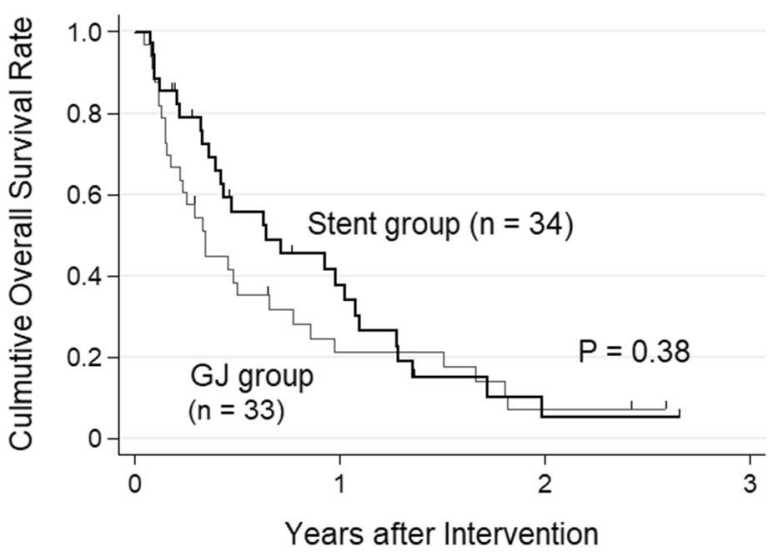

Fig. 1 Postoperative overall survival of patients with gastric outlet obstruction due to gastric cancer. a Patients who underwent endoscopic stenting or gastrojejunostomy (GJ) were analyzed by the Kaplan-Meyer method without risk adjustments. b Propensity score matching was performed to select the group of patients to undergo endoscopic stenting or GJ with similar characteristics

stent group, postoperative complications included one case of pneumonia with pleural effusion. This patient was discharged home. In gastrojejunostomy group, postoperative complications included two cases of pneumonia, one case of small bowel obstruction, one case of progressive renal dysfunction, one case of obstructive jaundice, one case of liver failure, and one case of incarceration of jejunum to port wound occurred in 7 patients. Three of them died in hospital. No significant differences were observed in GOOSS 3 achieved or the duration of GOOSS 3 between the two groups. Induction rates to chemotherapy were significantly less frequent in the stent group, whereas 1-year survival rates were higher in the stent group than in the gastrojejunostomy group. Thereafter, the two survival curves became similar (Fig. 1b).

\section{Discussion}

GOO is a life-threatening condition of advanced distal gastric cancer. This multicenter cohort study was undertaken to elucidate the background and outcomes of treatment for GOO due to gastric cancer. In the present study, stent therapy was selected for more advanced, more malnutritional, and more physically deteriorated patients with a shorter life expectancy over gastrojejunostomy. Therefore, treatment selection might be done depending on the patient status in the real clinical practice. The present results demonstrated that the technical and clinical success rates of stent therapy and gastrojejunostomy were similar to those in a high-volume center [8]. These results suggest that the interventions were performed by skilled doctors, although the present study included small- or mediumvolume centers in addition to high-volume centers. Postoperative morbidity was markedly less in the stent group than in the gastrojejunostomy group. An inferior postoperative food intake and shorter survival were observed in the stent group. We then performed propensity score matching, which is considered to reduce selection bias. The results obtained revealed no significant differences in food intake or survival between these two therapies, with less postoperative morbidity in the stent group. One-year survival rates were higher in the stent group than in the gastrojejunostomy group. This phenomenon may be related to less postoperative morbidity associated with stent placement because postoperative complications have been implicated in shorter postoperative survival times [20]. These findings suggest that stent therapy is potentially beneficial even for physically active patients with a longer life expectancy.

Previous studies have been conducted on treatment options for malignant GOO by including various types of cancers, such as pancreatic, biliary, duodenal, and stomach cancers [21]. Nevertheless, the type of obstruction may differ in each cancer. Furthermore, the prognosis of patients differs in each cancer. Therefore, treatment options need to be discussed according to the type of cancer being treated. Regarding GOO due to gastric cancer, we summarized previous studies in the supplemental table. Maetani et al. [4] initially described the comparative effectiveness of stent therapy with gastrojejunostomy for GOO due to gastric cancer on food intake and survival in 2005. In contrast, Kubota et al. [5] showed in 2007 that surgical stomach partitioning gastrojejunostomy was more advantageous for food intake and survival than stent therapy. Similar findings were reported by Arigami et al. [9] in 2016. In Korea in 2013, No et al. [6] found comparative food intakes between stent therapy and gastrojejunostomy, while survival times were longer with gastrojejunostomy. However, these studies did not address risk 
Table 3 Prognostic factors to achieve GOOSS 3 after interventions

\begin{tabular}{|c|c|c|c|c|}
\hline & \multicolumn{2}{|c|}{ Univariate analysis } & \multicolumn{2}{|c|}{ Multivariate analysis } \\
\hline & OR $(95 \% \mathrm{CI})$ & $p$ value & OR $(95 \% \mathrm{CI})$ & $p$ value \\
\hline Age & $0.9(0.5-1.4)$ & 0.59 & & \\
\hline Body mass index & $0.9(0.4-1.8)$ & 0.40 & & \\
\hline Macroscopic type IV & $0.1(0.05-0.4)$ & $<0.001$ & $0.12(0.03-0.5)$ & 0.005 \\
\hline Tumor location & $0.9(0.6-1.2)$ & 0.38 & & \\
\hline cT stage & $2.5(1.0-5.9)$ & 0.042 & $1.3(0.4-4.3)$ & 0.66 \\
\hline $\mathrm{cN}$ stage & $1.7(0.7-4.1)$ & 0.26 & & \\
\hline cM stage & $1.1(0.4-3.2)$ & 0.91 & & \\
\hline Preoperative GOOSS & $2.0(1.1-3.7)$ & 0.017 & $1.9(0.9-4.0)$ & 0.11 \\
\hline Severe heart disease & $0.1(0.0-1.8)$ & 0.12 & & \\
\hline Diabetes mellitus & $0.4(0.1-1.4)$ & 0.16 & & \\
\hline PS & $0.3(0.2-0.6)$ & $<0.001$ & $0.31(0.1-0.8)$ & 0.010 \\
\hline ASA-PS & $0.2(0.1-0.6)$ & 0.002 & $0.6(0.2-2.2)$ & 0.48 \\
\hline Serum albumin level & $2.0(0.9-4.4)$ & 0.053 & & \\
\hline Serum $\mathrm{Na}$ level & $8.3(2.3-30)$ & 0.001 & $3.8(0.7-21)$ & 0.13 \\
\hline Hemoglobin level & $1.3(0.6-3.1)$ & 0.55 & & \\
\hline White blood cell counts & $2.2(0.8-6.5)$ & 0.14 & & \\
\hline CEA & $0.6(0.3-1.0)$ & $\mathbf{0 . 0 3 2}$ & $0.5(0.3-1.1)$ & 0.072 \\
\hline CA19-9 & $0.6(0.3-1.1)$ & 0.11 & & \\
\hline Selection of stenting or GJ & $3.3(1.1-9.9)$ & 0.028 & $0.3(0.05-1.6)$ & 0.15 \\
\hline
\end{tabular}

Severe heart disease was defined as heart failure of New York Heart Association Class III or IV, or severe arrythmia requiring mechanical support [18]

Continuous variables were categorized according to the frequency of outcome variable, as Age for $<60$, $60-<70,70-<80$, and $\geq 80$; Body mass index for $\leq 20,20-\leq 25,25-\leq 30$, and $>30$; Serum albumin level $(\mathrm{g} / \mathrm{dL})$ for $<2.5,2.5-<3.5$, and $\geq 3.5$; Serum $\mathrm{Na}$ level $(\mathrm{mEq} / \mathrm{L})$ for $<126,126-130,131-135$, and $<135$; Hemoglobin level $(\mathrm{g} / \mathrm{dL})$ for $<8,8-<12$, and $\geq 12$; White blood cell counts $\left(/ \mathrm{mm}^{3}\right)$ for $4000-<10,000$ and others; CEA (ng/mL) for $<10,10-<100,100-<1000$, and $\geq 1000$; CA19-9 (U/mL) for $<100,100-<1000$, and $\geq 1000$

In ordinal variables, such as cT stage, $\mathrm{cN}$ stage, GOOSS, PS and ASA-PS, we did not binarize them and we used the original ordinal values, since the outcome variables increases when the ordinal values increased

$p$ values less than 0.05 are highlighted by bold letters

OR odds ratio, 95\% CI 95\% confidence interval, GOOSS gastric outlet obstruction scoring system, PS Performance status, ASA-PS American Anesthesiologists Association Physical Status, GJ Gastrojejunostomy, GOOSS is defined as 0 for no oral intake, 1 for liquids only, 2 for soft solids, 3 for a low residue or full diet adjustments, and, thus, a selection bias may be present between stent therapy and gastrojejunostomy. Fiori et al. [7] conducted a randomized controlled trial and reported that food intake and survival were similar between the two treatments. This is the only randomized controlled trial on this issue. However, the sample size was only 9 cases for each treatment, and, thus, it was underpowered. In 2015, Park et al. [8] performed a multivariate analysis and concluded that food intake was similar between the treatments, whereas survival was longer with gastrojejunostomy. In 2016, Park et al. [10] conducted propensity score matching for risk adjustments using a relatively large sample size and concluded that food intake and survival were similar between the treatments. On the other hand, Min [11] demonstrated that laparoscopic gastrojejunostomy resulted in better oral intake, better compliance to chemotherapy, and longer survival than stent therapy in a propensity score-matched analysis; however, the matching was so modest that 43 patients were selected from 58 patients in the stent group and 43 patients were unchanged in the gastrojejunostomy group. Furthermore, they did not show how baseline data were balanced between the treatment groups [11]. Therefore, it is questionable how much risk adjustment was made. Collectively, the study by Park [10] and the present study had a relatively large sample size with adequate risk adjustments and obtained similar results, suggesting reliability.

The present study revealed that the selection of stenting or gastrojejunostomy was not an independent risk factor for survival in the multivariate analysis, whereas macroscopic type IV and PRS were independent prognostic factors for survival. PRS is the key component of the E-PASS scoring 
Table 4 Prognostic factors for overall survival

\begin{tabular}{|c|c|c|c|c|}
\hline & \multicolumn{2}{|c|}{ Univariate analysis } & \multicolumn{2}{|c|}{ Multivariate analysis } \\
\hline & $\mathrm{HR}(95 \% \mathrm{CI})$ & $p$ value & $\mathrm{HR}(95 \% \mathrm{CI})$ & $p$ value \\
\hline Age & $1.5(1.2-1.8)$ & 0.001 & $1.3(1.0-1.7)$ & 0.10 \\
\hline Sex & $1.2(0.8-1.7)$ & 0.94 & & \\
\hline Body mass index & $1.0(0.8-1.4)$ & 0.32 & & \\
\hline Macroscopic type VI & $2.5(1.7-3.7)$ & $<0.001$ & $3.0(1.8-5.0)$ & $<0.001$ \\
\hline Tumor location & $1.7(1.2-2.4)$ & 0.005 & $1.1(0.7-1.7)$ & 0.67 \\
\hline cT stage & $0.8(0.6-1.1)$ & 0.28 & & \\
\hline $\mathrm{cN}$ stage & $0.8(0.5-1.1)$ & 0.11 & & \\
\hline cM stage & $1.1(0.7-1.6)$ & 0.29 & & \\
\hline Preoperative GOOSS & $0.8(0.7-1.0)$ & 0.027 & $0.9(0.8-1.1)$ & 0.54 \\
\hline PRS & $1.9(1.5-2.5)$ & $<0.001$ & $1.6(1.2-2.2)$ & 0.005 \\
\hline Biliary stent & $2.4(1.1-5.2)$ & 0.025 & $1.0(0.4-2.7)$ & 0.93 \\
\hline GPS & $1.6(1.3-2.0)$ & $<0.001$ & $1.3(0.9-1.8)$ & 0.16 \\
\hline Serum Na level & $0.7(0.5-0.9)$ & $\mathbf{0 . 0 3 3}$ & $0.8(0.6-1.2)$ & 0.41 \\
\hline Hemoglobin level & $1.4(1.0-2.0)$ & 0.031 & $1.4(1.0-2.1)$ & 0.085 \\
\hline White blood cell counts & $1.0(0.7-1.5)$ & 0.99 & & \\
\hline PNI & $2.0(1.4-2.9)$ & $<0.001$ & $1.4(0.8-2.3)$ & 0.21 \\
\hline CEA & $1.1(0.9-1.4)$ & 0.36 & & \\
\hline CA19-9 & $1.0(0.8-1.3)$ & 0.86 & & \\
\hline Selection of stenting or GJ & $0.6(0.4-0.8)$ & 0.001 & $0.6(0.4-1.0)$ & 0.069 \\
\hline
\end{tabular}

Continuous variables were categorized according to the frequency of outcome variable, as Age for $<75$, $75-\leq 80$, and $>80$; Body mass index for $\leq 20,20-\leq 25,25-\leq 30$, and $>30$; PRS for $<0.4,0.4-<0.6$, and $\geq 0.6$; serum Na level $(\mathrm{mEq} / \mathrm{L})$ for $<132$, 132-<140, and $\geq 140$; Hemoglobin $(\mathrm{g} / \mathrm{dL})$ for $8.6-11.5$ and others; White blood cell counts $\left(/ \mathrm{mm}^{3}\right)$ for $<4600$ and $\geq 4600$; PNI for $<35$ and $\geq 35$; CEA (ng/mL) for $<10,10-<100,100-<1000$, and $\geq 1000$; CA19-9 (U/mL) for $<100,100-<1000$, and $\geq 1000$

In ordinal variables, such as cT stage, $\mathrm{cN}$ stage, GOOSS and GPS, we did not binarize them and we used the original ordinal values, since the outcome variables increased when the ordinal values increased. On the other hand, tumor location was binarized according to the involvement of middle part

$p$ values less than 0.05 were highlighted by bold letters

$H R$ hazard ratio, 95\% CI 95\% confidence interval, GOOSS Gastric Outlet Obstruction Scoring system, PRS preoperative risk score, GPS Glasgow Prognostic Score, PNI Prognostic Nutritional Index, GJ Gastrojejunostomy

system along with the surgical stress score (SSS) and comprehensive risk score (CRS) [18]. These E-PASS scores have been shown to correlate with postoperative survival in various types of cancers [22-27]. Type IV cancer was identified as an independent risk factor for not only survival, but also postoperative food intake in multivariate analyses. Since type IV cancer is characterized by a thick gastric wall, it is conceivable that the mobility of the stomach may have been poor, resulting in reduced food intake regardless of the treatment type. Nevertheless, DGE was less common in the present study: less than $2 \%$ for both treatments. Therefore, stomach motility may not be the main reason for impaired food intake. The aggressiveness of this tumor may deteriorate both food intake and survival.

One of the major concerns regarding a metallic stent is the risk of gastrointestinal perforation after chemotherapy, particularly when using vascular endothelial growth factor inhibitors, such as bevacizumab and ramucirumab.
Regarding colonic cancer, Imbulgoda A et al. [28] reported that perforation rates after stenting were higher in patients undergoing chemotherapy with bevacizumab than in those undergoing chemotherapy without bevacizumab (20\%, 2/20 vs. 6\%, 3/47). In contrast, Lee et al. [29] showed that the perforation rate after stenting was not higher in the bevacizumab group than in the non-bevacizumab group for malignant colonic obstruction. Iwasaki et al. [30] reported that the use of an Ultraflex stent (odds ratio [OR] 6.81; 95\% confidence interval $[\mathrm{CI}] 1.54-30.00 ; p=0.011)$ and prior chemotherapy (OR 6.13; 95\% CI 1.46-25.70; $p=0.013$ ) correlated with stent-related complications in patients with malignant obstruction of the esophagus or gastric cardia. To the best of our knowledge, the risk of perforation after stent placement for GOO due to gastric cancer prior to or following chemotherapy has not yet been examined. In a case-series study, Endo et al. [31] performed stent placement on patients with GOO due to gastric cancer who had a 
Table 5 Baseline data after propensity score matching

\begin{tabular}{llll}
\hline & $\begin{array}{l}\text { Stent group } \\
(n=34)\end{array}$ & GJ group $(n=34)$ & $p$ value \\
\hline Age & $69(39-90)$ & $71(55-94)$ & 0.30 \\
Sex, \% male & $85 \%$ & $71 \%$ & 0.14 \\
Body mass index & $20(16-28)$ & $20(14-26)$ & $>0.99$ \\
GOOSS, 0: $1: 2: 3$ & $8: 14: 4: 6$ & $12: 10: 5: 7$ & 0.50 \\
PS, 0: $1: 2: 3$ & $6: 20: 7: 1$ & $4: 23: 4: 3$ & 0.49 \\
ASA-PS, $1: 2: 3$ & $6: 21: 7$ & $4: 26: 4$ & 0.42 \\
GPS, 0: 1: 2 & $4: 19: 11$ & $7: 13: 14$ & 0.32 \\
PNI & $37(21-47)$ & $37(26-48)$ & 0.16 \\
& $(n=29)$ & $(n=30)$ & \\
PRS & $0.46(0.13-1.0)$ & $0.52(0.26-0.92)$ & 0.36 \\
Macroscopic type & $21 \%(7 / 34)$ & $21 \%(7 / 34)$ & 1.0 \\
IV & & & \\
cT stage, 2: 3: 4 & $1: 17: 16$ & $1: 11: 22$ & 0.33 \\
cN (+) & $2: 27: 5$ & $6: 22: 6$ & 0.27 \\
Distant metastasis & $82 \%(28 / 34)$ & $76 \%(26 / 34)$ & 0.55 \\
Liver & $24 \%(8 / 34)$ & $32 \%(11 / 34)$ & 0.41 \\
Peritoneum & $56 \%(19 / 34)$ & $41 \%(15 / 34)$ & 0.32 \\
Distant lymph & $9 \%(3 / 34)$ & $38 \%(13 / 34)$ & $\mathbf{0 . 0 0 4}$ \\
nodes & & & \\
Other organs & $3 \%(1 / 34)$ & $3 \%(1 / 34)$ & 1.0 \\
CEA, ng/mL & $4.6(0.8-2640)$ & $7.3(0.8-1239)$ & 0.70 \\
\hline Da & &
\end{tabular}

Data for continuous variables are shown as medians (range)

$p$ values less than 0.05 are highlighted by bold letters

GJ gastrojejunostomy, GOOSS Gastric Outlet Obstruction Scoring system, 0 for no oral intake, 1 for liquids only, 2 for soft solids, 3 for a low residue or full diet, $P S$ performance status, ASA-PS American Anesthesiologists Association Physical Status, GPS Glasgow Prognostic Score, PNI Prognostic Nutritional Index, PRS Preoperative risk score good performance status, followed by chemotherapy in 11 patients. None of the patients were given bevacizumab or ramucirumab. Gastrointestinal perforation was not observed in these patients. Furthermore, five patients (45\%) underwent subsequent resection, four of whom achieved curative resections. Further studies are needed to ensure the feasibility and safety of chemotherapy for stent patients with GOO due to gastric cancer.

The main limitation of the present study is the relatively small sample size for the propensity score analysis, which was attributed to the rarity of GOO due to gastric cancer. Previous studies also have this limitation. Nevertheless, the present study analyzed multicenter data from a 1-year study period, exhibiting generalizability. Previous studies were generally single-center studies for a long study period. Another limitation is that we did not assess the nutritional status of patients after the interventions. Therefore, it remains unclear whether patients had a sufficiently dietary intake after interventions. Since this was a retrospective cohort study, many participating hospitals transferred patients to other hospitals. Difficulties were associated with obtaining sequential nutritional data. Nevertheless, since the present propensity score-matched analysis revealed equivalent postoperative survival between the treatments, the nutritional status may not have been significantly different.

In summary, we analyzed multicenter data on GOO due to gastric cancer in Japan. The propensity score-matched analysis revealed that endoscopic stent therapy conveyed less postoperative morbidity than and a similar postoperative food intake and equivalent survival to gastrojejunostomy. These results indicate the utility of stent therapy even for physically active patients.
Table 6 Outcome data after propensity score matching

\begin{tabular}{llll}
\hline & Stent group $(n=34)$ & GJ group $(n=34)$ & $p$ value \\
\hline Postoperative complications & $3 \%(1 / 33)$ & $21 \%(7 / 34)$ & $\mathbf{0 . 0 3}$ \\
In-hospital mortality & $9 \%(3 / 33)$ & $16 \%(5 / 30)$ & 0.37 \\
Length of stay, days $^{\mathrm{b}}$ & $27(5-147)$ & $21(7-102)$ & 0.94 \\
& $(n=34)$ & $(n=34)$ & \\
Clinical success $^{\mathrm{a}}$ & $81 \%(25 / 31)$ & $76 \%(26 / 34)$ & 0.68 \\
GOOSS 3 achieved & $97 \%(32 / 33)$ & $97 \%(33 / 34)$ & 0.98 \\
Duration of GOOSS 3, days & $118(12-616)$ & $112(11-876)$ & 0.62 \\
Induction of chemotherapy & $(n=24)$ & $(n=30)$ & \\
Subsequent resection & $24 \%(8 / 34)$ & $47 \%(16 / 34)$ & $\mathbf{0 . 0 4}$ \\
Median survival time $(\mathrm{M})^{\mathrm{c}}$ & $0 \%(0 / 33)$ & $6 \%(2 / 32)$ & 0.15 \\
& $7.8(4.0-16)$ & $4.0(1.8-10)$ & 0.38 \\
\hline
\end{tabular}

$p$ values less than 0.05 are highlighted by bold letters

GJ gastrojejunostomy, GOOSS Gastric Outlet Obstruction Scoring system, 0 for no oral intake, 1 for liquids only, 2 for soft solids, 3 for a low residue or full diet

${ }^{a}$ Indicates an improvement in GOOSS

${ }^{b}$ Data represent medians (range)

${ }^{\mathrm{c}}$ Data represent medians (interquartile range) 
Acknowledgements The authors wish to thank all the investigators who collected data at the participating hospitals. The authors are also grateful to Ms. Ryoko IIjima of the secretariat of the JGCA for her devoted secretarial work.

Funding This study was founded by the Japanese Gastric Cancer Association.

\section{Compliance with ethical standards}

Conflict of interest The authors declare that they have no conflict of interest associated with this manuscript.

Human rights statement All procedures followed were in accordance with the Ethical Guidelines for Medical and Health Research Involving Human Subjects in Japan.

Informed consent Informed consent from patients was waived, but information on this study was disclosed in the study website during data collection.

\section{References}

1. Bray F, Ferlay J, Soerjomataram I, Siegel RL, Torre LA, Jemal A. Global cancer statistics 2018: GLOBOCAN estimates of incidence and mortality worldwide for 36 cancers in 185 countries. CA Cancer J Clin. 2018;68:394-424.

2. Harada K, Lopez A, Shanbhag N, Badgwell B, Baba H, Ajani J. Recent advances in the management of gastric adenocarcinoma patients. F1000Res. 2018;7. pii: F1000 Faculty Rev-1365.

3. Yoshida K, Yamaguchi K, Okumura N, Tanahashi T, Kodera Y. Is conversion therapy possible in stage IV gastric cancer: the proposal of new biological categories of classification. Gastric Cancer. 2016;19:329-38.

4. Maetani I, Akatsuka S, Ikeda M, Tada T, Ukita T, Nakamura Y, et al. Self-expandable metallic stent placement for palliation in gastric outlet obstructions caused by gastric cancer: a comparison with surgical gastrojejunostomy. J Gastroenterol. 2005;40:932-7.

5. Kubota K, Kuroda J, Origuchi N, Kaminishi M, Isayama H, Kawabe T, et al. Stomach-partitioning gastrojejunostomy for gastroduodenal outlet obstruction. Arch Surg. 2007;142:607-11.

6. No JH, Kim SW, Lim CH, Kim JS, Cho YK, Park JM, et al. Longterm outcome of palliative therapy for gastric outlet obstruction caused by unresectable gastric cancer in patients with good performance status: endoscopic stenting versus surgery. Gastrointest Endosc. 2013;78:55-62.

7. Fiori E, Lamazza A, Demasi E, Decesare A, Schillaci A, Sterpetti AV. Endoscopic stenting for gastric outlet obstruction in patients with unresectable antro pyloric cancer. Systematic review of the literature and final results of a prospective study. The point of view of a surgical group. Am J Surg. 2013;206:210-7.

8. Park CH, Park JC, Kim EH, Chung H, An JY, Kim HI, et al. Impact of carcinomatosis and ascites status on long-term outcomes of palliative treatment for patients with gastric outlet obstruction caused by unresectable gastric cancer: stent placement versus palliative gastrojejunostomy. Gastrointest Endosc. 2015;81:321-32.

9. Arigami T, Uenosono Y, Ishigami S, Yanagita S, Okubo K, Uchikado Y, et al. Clinical impact of stomach-partitioning gastrojejunostomy with braun enteroenterostomy for patients with gastric outlet obstruction caused by unresectable gastric cancer. Anticancer Res. 2016;36:5431-6.
10. Park JH, Song HY, Yun SC, Yoo MW, Ryu MH, Kim JH, et al. Gastroduodenal stent placement versus surgical gastrojejunostomy for the palliation of gastric outlet obstructions in patients with unresectable gastric cancer: a propensity score-matched analysis. Eur Radiol. 2016;26:2436-45.

11. Min SH, Son SY, Jung DH, Lee CM, Ahn SH, Park DJ, et al. Laparoscopic gastrojejunostomy versus duodenal stenting in unresectable gastric cancer with gastric outlet obstruction. Ann Surg Treat Res. 2017;93:130-6.

12. The Ministry of Education, Culture, Sports, Science and Technology, and the Ministry of Health, Labour and Welfare of Japan. Ethical Guidelines for Medical and Health Research Involving Human Subjects. https://www.mhlw.go.jp/file/06-Seisakujou hou-10600000-Daijinkanboukouseikagakuka/0000080278.pdf. Accessed 22 Jan 2020

13. Adler DG, Baron TH. Endoscopic palliation of malignant gastric outlet obstruction using self-expanding metal stents: experience in 36 patients. Am J Gastroenterol. 2002;97:72-8.

14. Japanese Gastric Cancer Association. Japanese classification of gastric carcinoma: 3rd English edition. Gastric Cancer. 2011;14:101-12.

15. Clavien PA, Barkun J, de Oliveira ML, Vauthey JN, Dindo D, Schulick RD, et al. The Clavien-Dindo classification of surgical complications: five-year experience. Ann Surg. 2009;250:187-96.

16. Forrest LM, McMillan DC, McArdle CS, Angerson WJ, Dunlop DJ. Evaluation of cumulative prognostic scores based on the systemic inflammatory response in patients with inoperable nonsmall-cell lung cancer. Br J Cancer. 2003;89:1028-30.

17. Onodera T, Goseki N, Kosaki G. Prognostic nutritional index in gastrointestinal surgery of malnourished cancer patients. Nihon Geka Gakkai zasshi. 1984;85:1001-5 (in Japanese).

18. Haga Y, Ikei S, Ogawa M. Estimation of physiologic ability and surgical stress (E-PASS) as a new prediction scoring system for postoperative morbidity and mortality following elective gastrointestinal surgery. Surg Today. 1999;29:219-25.

19. Becker SO, Ichino A. Estimation of average treatment effects based on propensity scores. Stata J. 2002;2:358-77.

20. Shimada H, Fukagawa T, Haga Y, Oba K. Does postoperative morbidity worsen the oncological outcome after radical surgery for gastrointestinal cancers? A systematic review of the literature. Ann Gastroenterol Surg. 2017;1:11-23.

21. Minata MK, Bernardo WM, Rocha RS, Morita FH, Aquino JC, Cheng S, et al. Stents and surgical interventions in the palliation of gastric outlet obstruction: a systematic review. Endosc Int Open. 2016;4:E1158-E1170170.

22. Haga Y, Yagi Y, Ogawa M. Less-invasive surgery for gastric cancer prolongs survival in patients over 80 years of age. Surg Today. 1999;29:842-8.

23. Nanashima A, Abo T, Nonaka T, Fukuoka H, Hidaka S, Takeshita $\mathrm{H}$, et al. Prognosis of patients with hepatocellular carcinoma after hepatic resection: are elderly patients suitable for surgery? J Surg Oncol. 2011;104:284-91.

24. Ariake K, Ueno T, Takahashi M, Goto S, Sato S, Akada M, et al. E-PASS comprehensive risk score is a good predictor of postsurgical mortality from comorbid disease in elderly gastric cancer patients. J Surg Oncol. 2014;109:586-92.

25. Tominaga T, Takeshita H, Takagi K, Kunizaki M, To K, Abo T, et al. E-PASS score as a useful predictor of postoperative complications and mortality after colorectal surgery in elderly patients. Int J Colorectal Dis. 2016;31:217-25.

26. Yamamoto M, Saito H, Uejima C, Tanio A, Tada Y, Matsunaga T, et al. Estimation of physiological ability and surgical stress score is a useful prognostic indicator for elderly patients with colorectal cancer. Dig Surg. 2019. https://doi.org/10.1159/000497455.

27. Murakami Y, Saito H, Shimizu S, Kono Y, Shishido Y, Miyatani $\mathrm{K}$, et al. Evaluation of the estimation of physiologic ability and 
surgical stress score as a prognostic indicator for older patients with gastric cancer. Dig Surg. 2019. https://doi.org/10.1159/00049 7457.

28. Imbulgoda A, MacLean A, Heine J, Drolet S, Vickers MM. Colonic perforation with intraluminal stents and bevacizumab in advanced colorectal cancer: retrospective case series and literature review. Can J Surg. 2015;58:167-71.

29. Lee JH, Emelogu I, Kukreja K, Ali FS, Nogueras-Gonzalez G, Lum P, et al. Safety and efficacy of metal stents for malignant colonic obstruction in patients treated with bevacizumab. Gastrointest Endosc. 2019. https://doi.org/10.1016/j.gie.2019.02.016.

30. Iwasaki H, Mizushima T, Suzuki Y, Fukusada S, Kachi K, Ozeki

$\mathrm{T}$, et al. Factors that affect stent-related complications in patients with malignant obstruction of the esophagus or gastric cardia. Gut Liver. 2017;11:47-544.

31. Endo S, Nakagawa T, Konishi K, Ikenaga M, Ohta K, Nakashima $\mathrm{S}$, et al. Eleven patients with gastric cancer who received chemotherapy after stent placement for gastric outlet obstruction. Gan To Kagaku Ryoho. 2017;44:71-4 (In Japanese).

Publisher's Note Springer Nature remains neutral with regard to jurisdictional claims in published maps and institutional affiliations.

\section{Affiliations}

\section{Yoshio Haga ${ }^{1}$ (1) - Naoki Hiki ${ }^{2}$. Takahiro Kinoshita ${ }^{3} \cdot$ Toshiyasu Ojima $^{4} \cdot$ Yoshihiro Nabeya $^{5}$. Shirou Kuwabara ${ }^{6}$. Yasuyuki Seto ${ }^{7} \cdot$ Kazuhito Yajima $^{8} \cdot$ Hiroya Takeuchi $^{9} \cdot$ Kazuhiro Yoshida $^{10} \cdot$ Yasuhiro Kodera $^{11} \cdot$ Yoshiyuki Fujiwara $^{12}$. Hideo Baba ${ }^{13}$}

1 Department of Surgery, Japan Community Healthcare Organization Amakusa Central General Hospital, 101 Higashi-machi, Amakusa-shi 8630033, Japan

2 Department of Gastroenterological Surgery, Cancer Institute Hospital of the Japanese Foundation for Cancer Research, Tokyo, Japan

3 Gastric Surgery Division, National Cancer Center Hospital East, Kashiwa, Japan

4 Second Department of Surgery, School of Medicine, Wakayama Medical University, Wakayama, Japan

5 Division of Esophago-Gastrointestinal Surgery, Chiba Cancer Center, Chiba, Japan

6 Department of Digestive Surgery, Niigata City General Hospital, Niigata, Japan

7 Department of Gastrointestinal Surgery, Graduate School of Medicine, University of Tokyo, Tokyo, Japan
8 Department of Surgery, Sainokuni Higashiomiya Medical Center, Saitamashi, Japan

9 Department of Surgery, Hamamatsu University School of Medicine, Hamamatsu, Japan

10 Department of Surgical Oncology, Gifu University Graduate School of Medicine, Gifu, Japan

11 Department of Gastroenterological Surgery, Nagoya University Graduate School of Medicine, Nagoya, Japan

12 Department, of Surgery, School of Medicine, Tottori University Faculty of Medicine, Yonago, Japan

13 Department of Gastroenterological Surgery, Graduate School of Medical Sciences, Kumamoto University, Kumamoto, Japan 